\title{
COMPARISON OF DIFFERENT TREATMENTS OF THE RADIATION TRANSFER IN MODEL CALCULATIONS OF PLANETARY NEBULAE
}

\author{
G. BÄSSGEN, M. BÄSSGEN, M. GREWING \\ Astronomisches Institut Tübingen (FRG)
}

\begin{abstract}
A method of Radiation Transfer treatment (Global Shell Model (GSM)) has been developed, different from the On the Spot Approximation (OSA), taking into account all diffuse reemission from radiative recombination of hydrogen and helium in the nebula itself. For optically thick and optically thin nebulae the method automatically reproduces the On the Spot Approximation and the models without any Radiation Transfer, respectively.

In a model nebula consisting of $\mathrm{N}$ concentric spherical shells the diffuse radiation field at any one point is calculated along several characteristic rays through the nebula, including the outer parts. Diffuse reemission from these outer regions becomes important, when the density increases with distance from the central star.

The calculation of the ionisation and thermal balance is an iterative process.

A grid of model nebulae has been calculated in order to compare the performance of the OSA, where the diffuse reemission is calculated by the source function $\varepsilon_{\lambda} / x_{\lambda}$, and our Global Shell Model.

The grid covers a wide range of input parameters such as the temperature and luminosity of the nuclei and also the density distribution in the nebulae themselves.

In overall optically thin nebulae the On the Spot Approximation overestimates the number of reemitted $\mathrm{H}-\mathrm{Ly}$-photons with an energy close to $13.6 \mathrm{eV}$ and thereby lowers the temperature in the whole nebula. Most emission line strengths turn out weaker than in the GSM.

For nebulae with moderate optical thickness the situation is different. In the innermost optically thin regions, the OSA again overestimates the diffuse radiation and lowers the temperature, emission lines turn out weaker than in the GSM. As the optical thickness increases with increasing radius, the temperature curves cross each other and the situation changes. The temperature curves cross at the same distance from the nucleus where the overestimation of the diffuse radiation becomes an underestimation in the OSA. The extent to which the diffuse radiation is underestimated depends very much on the density. It is larger for objects with a large density gradient. The resulting integrated emission line strengths depend critically on such temperature differences.

For real nebulae one has to check on a case by case basis whether a theoretical modelling without any radiation transfer treatment or with the 'On the Spot Approximation' are justified or whether the more elaborate treatment described here should be applied.
\end{abstract}

